This is a postprint version of the following published document:

Rodríguez-Millán, M.; Ito, T.; Loya, J. A.; Olmedo, A.; Miguélez, M. H. (2016).

Development of numerical model for ballistic resistance evaluation of combat helmet and experimental validation. En: Materials \& Design, v. 110, pp. 391-403.

DOI: $10.1016 /$ j.matdes.2016.08.015
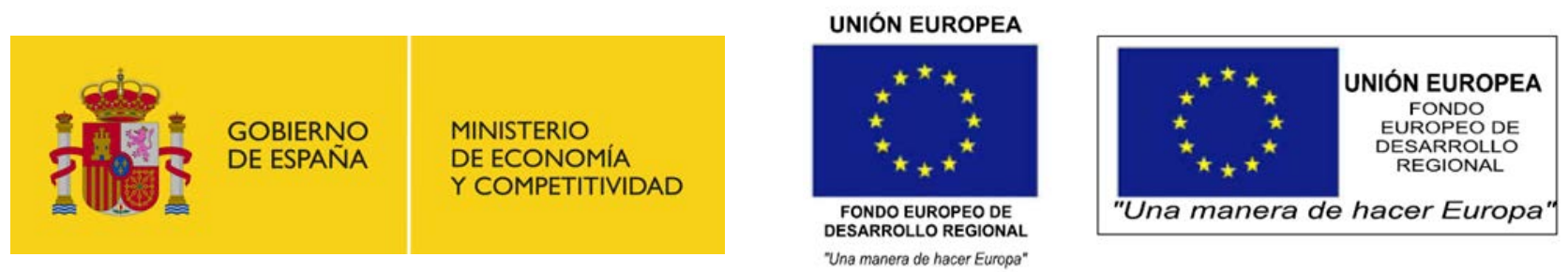

Project RTC-2015-3887-8

CC Elsevier 2016

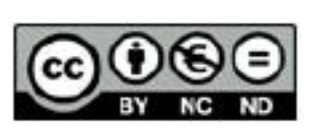

This work is licensed under a Creative Commons Attribution-NonCommercialNoDerivatives 4.0 International License. 


\title{
Development of numerical model for ballistic resistance evaluation of combat helmet and experimental validation
}

\author{
M. Rodríguez-Millán ${ }^{\mathrm{a}, *}$, T. Ito $^{\mathrm{b}}$, J.A. Loya ${ }^{\mathrm{b}}$, A. Olmedo $^{\mathrm{c}}$, M.H. Miguélez $^{\mathrm{a}}$ \\ a Department of Mechanical Engineering, University Carlos III of Madrid, Avda. de la Universidad 30, 28911 Leganés, Madrid, Spain \\ b Department of Continuum Mechanics and Structural Analysis, University Carlos III of Madrid, Avda. de la Universidad 30, 28911 Leganés, Madrid, Spain \\ c FECSA Company, Calle de Acacias 3, 28703 San Sebastián de los Reyes, Madrid, Spain
}

H I G H L I G H T S

- Numerical models of impact on aramid composite plates and combat helmet were developed.

- Experimental tests were carried out ac cording to standards used by manufac turers.

- Good accuracy between predictions and experiments was observed proving the ability of the models developed as a de sign tool
Combat helmet

Numerical modeling

Ballistic impact

Back face deformation

$\mathrm{V}_{50}$ velocity
G R A P H I C A L A B S T R A C T

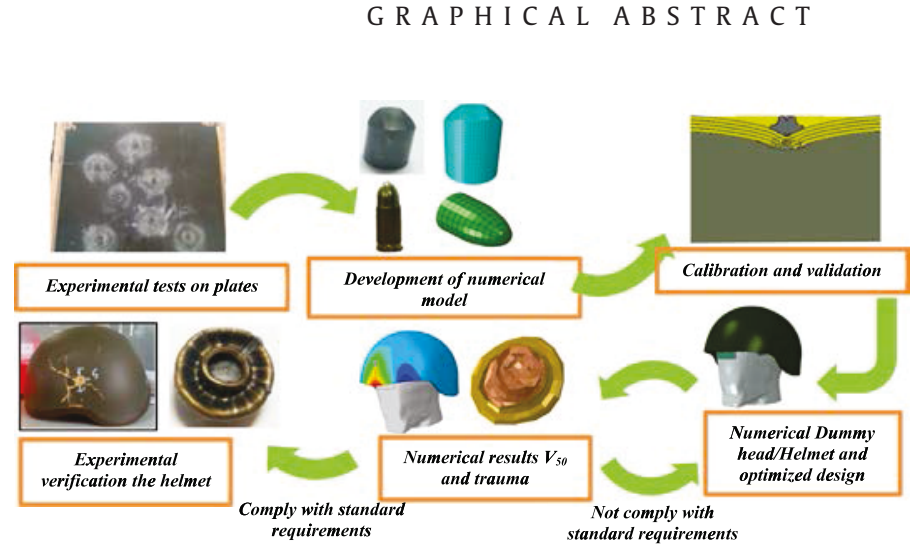

\begin{abstract}
A b s t r a c t
Modern designing process of combat helmets requires both numerical modeling and experimental validation in order to achieve exigent requirements combining impact resistance and reasonable weight. In this work a finite element model of a combat helmet is presented. Mechanical behaviour of the shell aramid composite under im pact conditions was analyzed from experimental Fragment Simulating Projectile (FSP) and Full Metal Jacketed (FMJ) impact tests on aramid flat plates. Numerical modeling based on finite elements method was used to sim ulate both impacts in simple plates of the composite and also the simulation of ballistic impact involving real am munition and the complex geometry of the helmet including inner foam. Experimental work involving impact tests on composite plates and also ballistic test on the helmet with a dummy provided real data for comparison with models predictions and proved the accuracy of the numerical models developed.
\end{abstract}

\section{Introduction}

Given the recent rise in terrorism, civil and international con flicts, the number of people afflicted with war related Traumatic

* Corresponding author.

E-mail addresses: mrmillan@ing.uc3m.es (M. Rodríguez-Millán), aolmedo@fecsa.net (A. Olmedo).
Brain Injuries (TBI) is increasing. In this sense, TBI is most often related to their exposure to blast and ballistic threats, such as those produced due to impact of shrapnel, shell fragments, and bullets.

In order to minimize the morbidity and mortality resulting from bal listic head injuries, that the role of personal protective equipment is cru cial, especially in the case of the combat helmets. Modern designing process requires both numerical modeling and experimental validation 


$\begin{array}{ll}\text { Nomenclature } \\ \text { ACH } & \text { Advanced Combat Helmet } \\ \text { BABT } & \text { Behind Armor Blunt Trauma } \\ \text { BFD } & \text { back face deformation } \\ \text { BHBT } & \text { Behind Helmet Blunt Trauma } \\ \text { DIC } & \text { Digital Image Correlation } \\ \text { FE } & \text { finite element } \\ \text { FEA } & \text { Finite Element Analysis } \\ \text { FMJ } & \text { Full Metal Jacketed } \\ \text { FSP } & \text { Fragment Simulating Projectile } \\ \text { ICP } & \text { Intracranial Pressure } \\ \text { NIJ } & \text { National Institute of Justice } \\ \text { PASGT } & \text { Personal Armor System for Ground Troops } \\ \text { STANAG } & \text { Standardization Agreement } \\ \text { TBI } & \text { Traumatic Brain Injuries }\end{array}$

in order to achieve exigent requirements combining impact resistance and reasonable weight.

Designing of combat helmets have evolved considerably over the years from steel helmet at the beginning of 20th century to the helmet made of composite materials at the second part of 20th century, as Per sonal Armor System for Ground Troops (PASGT) or Advanced Combat Helmet (ACH) helmets [1].

Protective structures offering penetration resistance against incident high energy projectiles based on aramid composites have been com monly widely studied in the literature [2 6]. The most used aramid composite, Kevlar, presents excellent mechanical properties, mainly high strength, high modulus, and high strength to weight ratio.

The helmet's protective capabilities are commonly evaluated in terms of two parameters: the impact velocity (in general $V_{50}$ velocity) and the back face deformation (BFD). The $V_{50}$, also known as the ballistic limit velocity, is defined as the required velocity of the projectile to per forate the target $50 \%$ of the times [12]. A minimum of two partial and two complete penetration velocities should be considered to determine the $V_{50}$ velocity [7]. However, even when the helmet is able to stop the projectile, other serious injuries can occur such as Behind Helmet Blunt Trauma (BHBT). This trauma is caused due to no penetrating ballistic impacts since the energy deposited in the helmet by the retarded pro jectile may be transferred through the interior foams. BHBT is often as sociated with helmet back face deformation (BFD) [8].

The analysis of BFD requires a methodology to measure the trauma: a block of clay or gelatin is commonly used to get this goal. In some cases, a piece of clay is embedded in a ballistic dummy head. However, in the literature, it is also possible to found some experimental and nu merical studies with a human skull [9 11 ] or numerical head models such as [12 15].

The experimental work of Sarron et al. [10] is one of the earliest pa pers focused on the analysis of BFD. The authors performed non pene trating ballistic lateral impacts of a $9 \mathrm{~mm}$ projectile on silicone filled dry skull protected by a polyethylene plate. This work has become a ref erence for further studies in the literature. Hisley et al. [16] quantified the helmet BFD and correlated it to BC injury. For this purpose, they used Digital Image Correlation (DIC) technique which allowed measur ing dynamic displacements, for further calculation of deformation, ve locity, and acceleration rates. Tham et al. [17] carried out experiments and simulations on the ballistic impact of a Kevlar helmet using spheri cal projectiles of $11.9 \mathrm{~g}$ at $205 \mathrm{~m} / \mathrm{s}$. Moreover, they developed numerical simulations with other type of projectiles; Full Metal Jacketed (FMJ) $9 \mathrm{~mm}$ bullet at $358 \mathrm{~m} / \mathrm{s}$ and Fragment Simulating Projectile (FPS); to ob tain the $\mathrm{V}_{50}$ velocity. Tan et al. [18] carried out both experiments and numerical simulations of frontal and lateral ballistic impacts on a Hybrid
III headform equipped with Advanced Combat Helmet (ACH) using $11.9 \mathrm{~g}$ spherical steel projectile at $220 \mathrm{~m} / \mathrm{s}$. Their analysis included quan titative parameters, such as head accelerations, helmet damage and de flection. Recently, Rafaels et al. [11] developed experimental analysis of seven postmortem human head/neck specimens wearing a ballistic pro tective helmet exposed to no perforating impact, using a FMJ $9 \mathrm{~mm}$ bul let with velocities of $400460 \mathrm{~m} / \mathrm{s}$. They showed that the contact and fracture phenomena were generally different for Behind Armor Blunt Trauma (BABT) than those for direct penetrating trauma classically de scribed in the literature.

Few experimental works have been published in the literature as it is shown in the aforementioned studies. The complexity of the ex perimental tests, involving expensive experimental devices such as ballistic gas gun, justifies the use of numerical models for simulation of BHBT.

In the early 2000s, Van Hoof et al. [19] performed ballistic impact tests on panels based on woven Kevlar composite using different types of projectiles. It was concluded that the relationship between the maximum backplane displacement and the impact energy is nearly linear within the range of impact energies considered in the work. Aare and Kleiven [20] determined numerically the effects of shell stiffness and impact angles on the level of the transferred load to the modeled human head. The main conclusion was that the helmet shell deflections should not exceed the initial gap between the helmet shell and the head in order to prevent the rear effect. The results were validated using the experimental data from shooting tests presented in [21].

In 2010, Lee et al. [22] developed numerical simulations in order to compare the protection efficiency of PASGT against head injury due to FSP and FMJ bullet when considering different interior cushioning sys tems. They concluded that the helmet together with its interior strap of fered a good protection against small fragments but behaved poorer against large projectile rounds. Tan et al. [18] performed experimental ballistic tests and finite element (FE) simulations in ACH using spherical bullets in order to check the effectiveness of its interior cushioning sys tems. Another study of these authors, Tse et al. [12,23] developed a de tailed numerical model of human head in order to obtain the Intracranial Pressure (ICP) in the brain and the maximum helmet de flection using FMJ bullet. A recent study was carried out by Li et al. [8]. They obtained numerically the BFD recorded by the clay using the dummy head/clay and by the helmet using FMJ bullet for different im pact locations.

A summary of the main results obtained in the aforementioned works is summarized in Table 1 including projectile and protection type, velocity range and BFD obtained.

Innovative helmet designs require ballistic testing of the structural elements constituting the personal protection. Ballistic impact tests used by the manufacturers are defined in the standards NIJ Standard 0106.01 for ballistic helmets [24] and Standardization Agreement (STANAG) 2920: Ballistic Test Method for personal armor materials and combat clothing [25], being the most used by the manufacturers.

STANAG 2920 [25] uses chisel nosed fragment simulating projec tiles (FSP) for proof testing. The $1.1 \mathrm{~g}$ ( 17 grain) FSP with 0.22 caliber (5.5 mm diameter) is the most frequently used. This standard is used to calculate the $V_{50}$ velocity of a specimen. In the simplest approach, $\mathrm{V}_{50}$ could be determined by averaging six projectile striking velocities that include three lowest velocities that resulted in complete penetra tion and the three highest velocities that resulted in a partial penetra tion as it is explained in [17].

NIJ STD 0106.01[10] establishes the performance requirements and methods of testing helmets intended to protect the wearer against gun fire. The standard classifies ballistic helmets into three types depending on the level of performance. This study focuses on Type II which states ballistic impact performance requirements for a helmet when impacted by $9 \mathrm{~mm}$ Full Metal Jacketed (FMJ) bullet weighing $8 \mathrm{~g}$ (124 grain) from the front, side, rear and top of the combat helmet at $425 \pm 15 \mathrm{~m} / \mathrm{s}$. A pad system and headform with ballistic clay Roma Plastilina No. 1 are used 
Table 1

Summary of some works in the literature obtaining BFD through experimental and numerical approaches for different configurations [8,12,15,18-23]

\begin{tabular}{|c|c|c|c|c|c|c|}
\hline Material & Thickness (mm) & Projectile & $\mathrm{V}_{0}(\mathrm{~m} / \mathrm{s})$ & $\mathrm{BFD}(\mathrm{mm})$ & Type of analysis & Reference \\
\hline Aramid laminate & $6.94-8.56$ & FMJ $8 \mathrm{~g}$ & $351-362$ & $28-35$ & Experimental & {$[20,21]$} \\
\hline Aramid laminate & 9.5 & FSP $1.1 \mathrm{~g}$ & 586 & 14 & Numerical & [19] \\
\hline Aramid laminate & 9.15 & FSP $1.1 \mathrm{~g}$ & 610 & 8.8 & Numerical & [22] \\
\hline Aramid laminate & 9.15 & FMJ $8 \mathrm{~g}$ & 358 & 17.3 & Numerical & [22] \\
\hline Aramid laminate & No data specified & FMJ $8 \mathrm{~g}$ & $370 \pm 7.6$ & $\sim 40$ & Experimental & [16] \\
\hline Aramid laminate & 9.15 & Sphere $11.9 \mathrm{~g}$ & 205 & 12.6 & Experimental \&Numerical & [18] \\
\hline Aramid laminate & 9.15 & FMJ $8 \mathrm{~g}$ & 358 & 10.9 & Numerical & {$[12,23]$} \\
\hline Aramid laminate & No data specified & FMJ $8 \mathrm{~g}$ & 370 & $\begin{array}{l}20.3 \text { (Frontal) } \\
25.6 \text { (Lateral) }\end{array}$ & Numerical & [8] \\
\hline
\end{tabular}

to measure the helmet back face deformation. The helmet pad was used in the interior foam cushioning system.

As it was commented previously, numerical modeling provides an invaluable tool for characterizing the physics of the problem in terms of relevant mechanical variables such as stress, strain, and acceleration. Therefore, models are a very useful virtual testing tool that helps to know of the significant variables that contribute to reducing BFD would enhance the selection of new and appropriate materials and a proper design, in order to improve protection from blunt trauma and possible weight reduction. On the other hand, the creation of complex models combining the mechanical response of the helmet and the bio mechanical effect of the head require the development of previous de tailed model of the helmet. The accuracy of the numerical predictions depends on the selection of constitutive and failure models and the proper calibration of its parameters in conditions similar to those occur ring during impact events.

In this paper, a numerical finite element model was developed in order to predict the response of a combat helmet subjected to ballistic impact. The model parameters were calibrated through experimental tests on plates performed according to STANAG 2920 and NIJ STD 0106.01 in order to obtain material properties. Numerical results for the helmet under ballistic impact were compared with real impact tests showing the accuracy of the model and its suitability to be used as a designing tool. The experimental work carried out to obtain helmet material properties and ballistic performance of the real helmet is pre sented in the second section. Numerical modeling including both the simplified models of the plate and the complex model representing the real helmet is presented in the third section. Experimental and nu merical results are presented and discussed in the fourth section and fi nally conclusions are stated in the last section.

\section{Experimental work}

In this section experimental devices and the tests are described Firstly, the ballistic tests on aramid plates are presented. The aim is char acterizing the materials those are used in the helmet resistant structure. Secondly the impact tests on the helmet carried out according to stan dards are described.

\subsection{Impact tests on aramid composite plates}

Experimental tests were carried out according to standards (STANAG 2920 and NIJ STD 0106) with the aim of characterizing the mechanical behaviour of the aramid composite under ballistic impact. These experimental results were used for the calibration of a numerical model developed to simulate the combat helmet behaviour under bal listic impacts. The dimensions of aramid composite specimens consid ered were $400 \times 400 \mathrm{~mm}^{2}$, firmly clamped on the edges. The areal density is $8.86 \mathrm{~kg} / \mathrm{m}^{2}$, representative of the helmet laminate configura tion. The aramid composite specimens contain more than 20 laminate layers and are molded from Kevlar 29/polyvinyl butyral phenolic com posite. Impact tests were conducted using a pneumatic gas gun.
In order to obtain the $\mathrm{V}_{50}$ velocity, the STANAG 2920 protocol is used. The target was impacted by a Fragment Simulating Projectile (FSP) with 0.22 caliber ( $5.5 \mathrm{~mm}$ diameter). The initial and residual ve locities were measured by two laser photodetectors.

Seven shots were conducted with $1.1 \mathrm{~g}$ FSP based on STANAG 2920 protocol with a velocity of $650 \pm 40 \mathrm{~m} / \mathrm{s}$. The result of $\mathrm{V}_{50}$ after seven shoots (Table 2) was $686.6 \mathrm{~m} / \mathrm{s}$ for aramid composite plates, higher than the obtained by other researchers for aramid composite plates ( $586 \mathrm{~m} / \mathrm{s}$ [28], $610 \mathrm{~m} / \mathrm{s}$ [17]) and closer to the value $680 \mathrm{~m} / \mathrm{s}$ obtained by Colakoglu et al. [29]. Fig. 1 shows the final stage of the plate penetra tion for the front and back side.

The NIJ STD 0106.01 protocol was used to obtain the maximum de formation along its normal direction. For such purpose, a block of clay was placed behind the target. Seven shots were conducted with $9 \mathrm{~mm}$ full metal jacket (FMJ) bullet, with nominal mass of $8.0 \mathrm{~g}$ (124 grain) and velocity of $425 \pm 15 \mathrm{~m} / \mathrm{s}$. Fig. 3 shows the final stage of the penetra tion for front and back side.

Experimentally, the values of back face deformation (BFD) obtained are summarized in Fig. 2 for impact velocity $V_{0}=425 \mathrm{~m} / \mathrm{s}$. The average experimental BFD obtained was $21.8 \pm 1.35 \mathrm{~mm}$. An increase in BFD with the tests is observed, possibly, due to the accumulated delamina tion of laminate.

\subsection{Impact tests on combat helmets}

Experimental tests on combat helmets were carried out according to standards (STANAG 2920 and NIJ STD 0106).

The STANAG 2920 protocol was used to obtain the $V_{50}$ velocity of the combat helmet shell. The combat helmet shell without headform was impacted by a Fragment Simulating Projectile (FSP) with 0.22 caliber (5.5 mm diameter) [27]. Seven shoots in different places of the helmet were performed covering a wide range of impact veloci ties ( $663.6 \mathrm{~m} / \mathrm{s} \leq \mathrm{V}_{0} \leq 732 \mathrm{~m} / \mathrm{s}$ ) (Fig. 3), resulting in an experimental $\mathrm{V}_{50}$ of $697 \mathrm{~m} / \mathrm{s}$ (higher than calculated by other researchers $[17,38,39]$ ).

The analysis of BFD was carried out according to NIJ STD 0106.01 protocol. The experimental setup consisted of the combat helmet shell, a pad system, and a fixed headform with ballistic clay Roma Plastilina No. 1. The BFD was measured by the clay.

Table 2

Experimental tests for $\mathrm{V}_{50}$ velocity on plates.

\begin{tabular}{llll}
\hline Impact & Impact velocity $(\mathrm{m} / \mathrm{s})$ & Complete penetration & Partial penetration \\
\hline 1 & 696.3 & $\mathrm{X}$ & \\
2 & 691.5 & $\mathrm{X}$ & $\mathrm{X}$ \\
3 & 658.5 & & $\mathrm{X}$ \\
4 & 665.6 & & $\mathrm{X}$ \\
5 & 692.2 & $\mathrm{X}$ & $\mathrm{X}$ \\
6 & 703.7 & & \\
7 & 670.2 & 686.6 & \\
Ballistic limit $(\mathrm{m} / \mathrm{s})$ & &
\end{tabular}




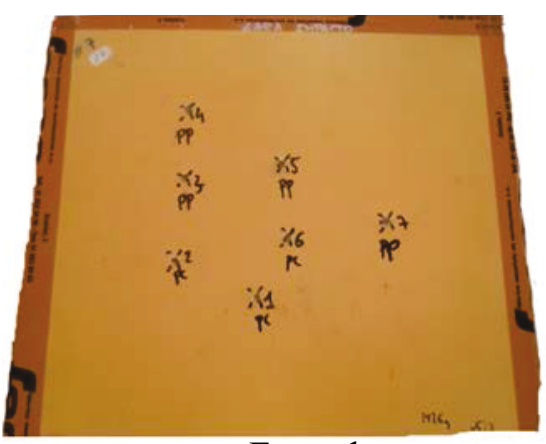

Frontal

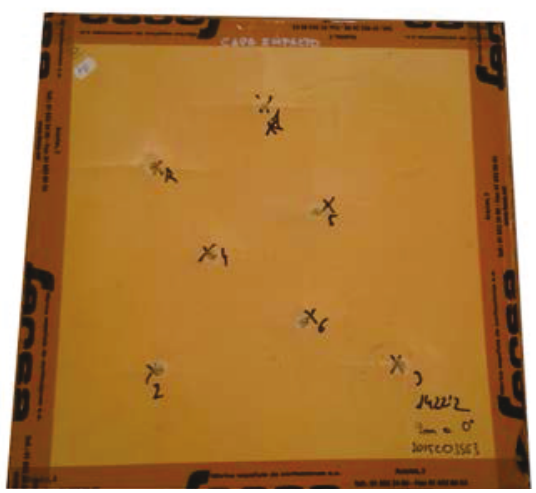

Frontal

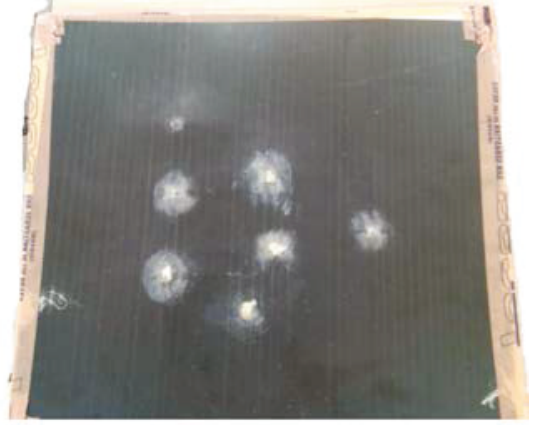

Back

a)

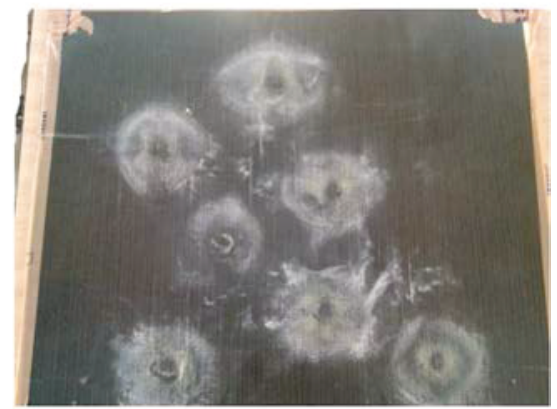

Back

b)

Fig. 1. Final stage of the test conducted using a) STANAG 2920 protocol and the b) NIJ Standard 0106.01 protocol for seven shots.

Five shots (front, sides, rear and top of the combat helmet) were conducted with $9 \mathrm{~mm}$ full metal jacket bullet, with nominal mass of $8.0 \mathrm{~g}$ (124 grain) and velocity of $425 \pm 15 \mathrm{~m} / \mathrm{s}$. The values of BFD obtain ed during impact on front, top, back, left and right sides were, respec tively, $12,11,9,6$, and $11 \mathrm{~mm}$.

\section{Numerical modeling}

In this section numerical models are presented. As it was ex plained previously two different models were developed. Firstly,

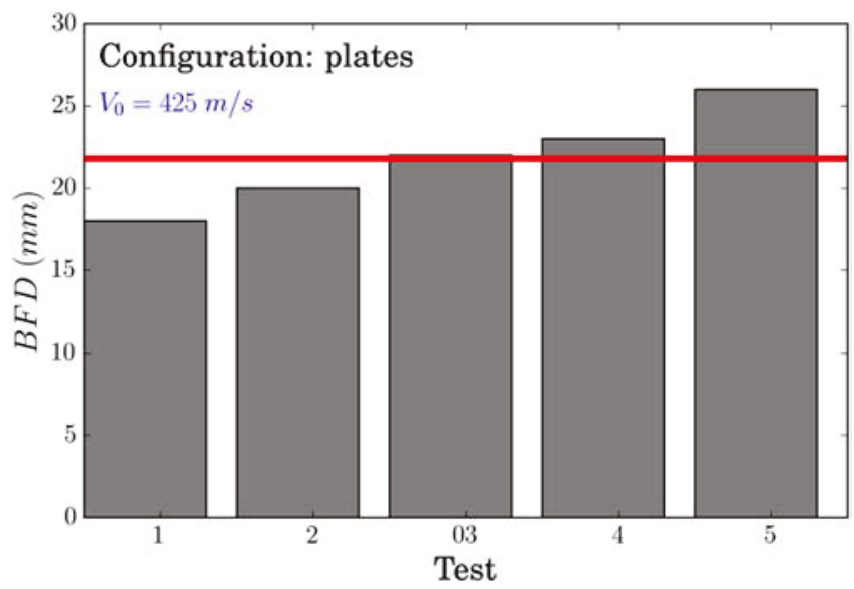

Fig. 2. Experimental values of back face deformation (BFD) for aramid composite plates. Red line: average value of BFD $=21.8 \pm 1.35 \mathrm{~mm}$. (For interpretation of the references to color in this figure legend, the reader is referred to the web version of this article.) the model of the simplified configuration for ballistic tests on aramid plates is developed with the aim of calibrating the material parame ters required for the accurate simulation of the helmet behaviour. Secondly the real helmet is modeled including inner foams. Common elements of both models are described at the beginning of the sec tion and the specific characteristics of each model are explained in detail.

Both the plate and the combat helmet models were developed using the finite element (FE) commercial code ABAQUS/Explicit based on a Lagrangian approach, allowing efficient reproduction of the dynamic loading process.

The contact between the projectiles and the plates was defined with penalty contact algorithm and hard contact model according to ABAQUS code user manual [30]. The "hard contact" option allows adjusting automatically the stiffness, generated by the "penalty con tact algorithm", to minimize penetration without adversely affecting the time increment. Concerning frictional effects, it is assumed a dy namic frictional coefficient $\mu$ equal to 0.3 between steel and compos ite. For the cases requiring the simulation of clay, the coefficient of friction between the metal and Roma Plastilina No. 1 was equal to 0.193 according to [31].

The specific characteristics of the FE models are described in the following paragraphs. Firstly, the modeling scheme of the projectiles and the composite plates is presented in the first and second subsec tions. The use of ballistic clay is required in the standards as a back element recording the trauma, thus the modeling of this component is included in the third subsection. The model of composite plates in presented in the fourth subsection together with its validation, it is worth to note the importance of this intermediate step allowing check the validity of the hypothesis formulated. Finally, in the fifth subsection, the complex model of the combat helmet is described in cluding inner protective foams. 


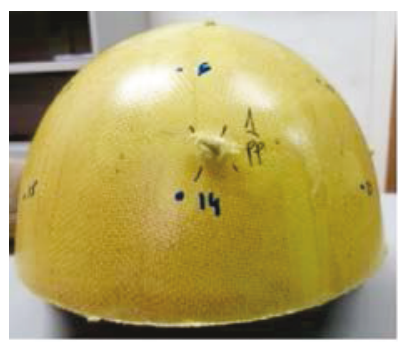

a) Front side

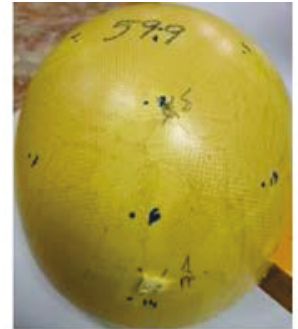

b) Top side

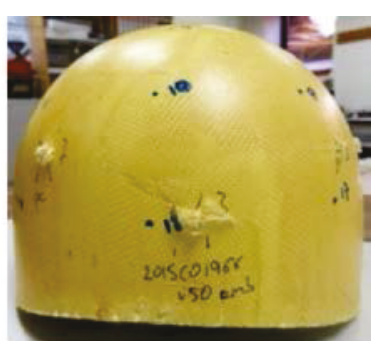

c) Back side

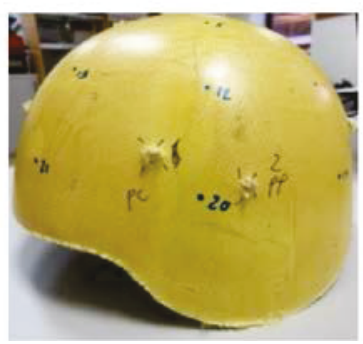

d) Left side

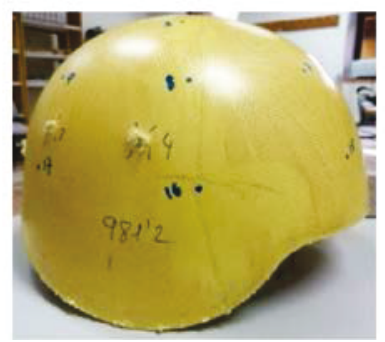

e) Right side

Fig. 3. Final stage of the STANAG 2920 tests on combat helmets.

\subsection{Projectiles}

\subsubsection{Fragment Simulating Projectile (FSP)}

The projectile used in the simulation was a $1.1 \mathrm{~g}$ FSP defined in STANAG 2920 protocol. The FSP was made of AISI 4340 steel and was meshed with C3D6 elements and average size $0.3 \mathrm{~mm}$ (see real FSP and model in Fig. 4).

The thermoviscoplastic material behaviour of the FSP was defined using the Johnson Cook (JC) model [35]. The JC model is generally pre implemented in finite element (FE) codes, including ABAQUS/Ex plicit. This hardening law is defined by Eqs. (1) and (2). The first term defines strain hardening $\left(\bar{\varepsilon}^{p}\right)$, the second strain rate sensitivity $\left(\dot{\bar{\varepsilon}}^{p}\right)$ and the third one is related to the thermal softening $(\Theta)$ :

$$
\begin{aligned}
& \bar{\sigma}\left(\overline{\bar{\varepsilon}}^{p}, \dot{\bar{\varepsilon}}^{p}, T\right) \quad\left[A+B\left(\bar{\varepsilon}^{p}\right)^{n}\right]\left[1+C \ln \left(\frac{\dot{\bar{\varepsilon}}^{p}}{\dot{\bar{\varepsilon}}_{0}}\right)\right]\left[1-\Theta^{m}\right] \\
& \Theta \frac{T-T_{0}}{T_{m}-T_{0}}
\end{aligned}
$$

where $\bar{\sigma}$ is the effective flow stress, $\bar{\varepsilon}^{p}$ is the accumulated plastic strain, $\dot{\bar{\varepsilon}}^{p}$ is the equivalent plastic strain rate and $T$ is the absolute temperature.
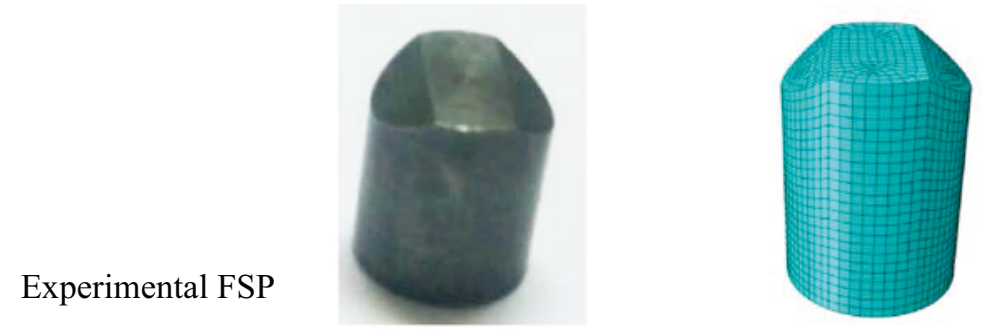

Numerical FSP
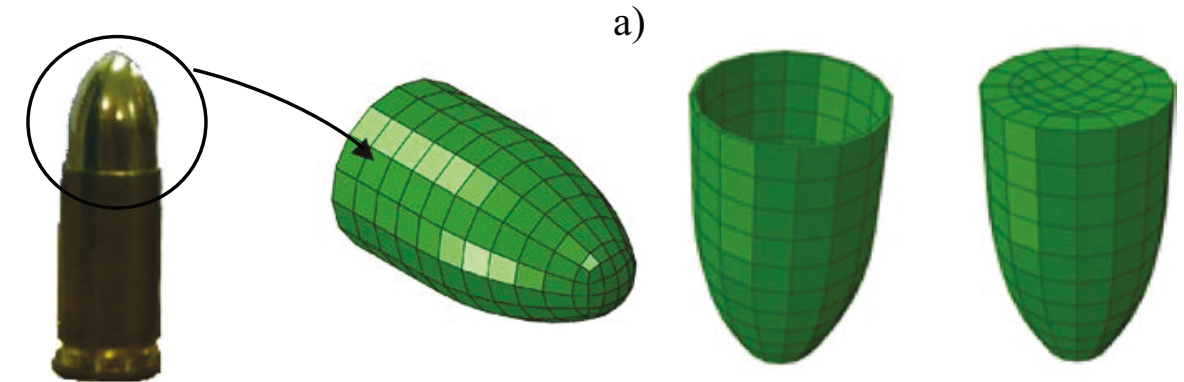

Experimental FMJ

Numerical FMJ

Cartridge brass

Lead core

b)

Fig. 4. Experimental and numerical model of: a) Fragment Simulating Projectile (FSP) and b) Full Metal Jacket (FMJ). 
Table 3

Mechanical properties of AISI 4340 [36].

\begin{tabular}{|c|c|c|c|c|c|c|c|}
\hline \multicolumn{2}{|c|}{ Elasticity } & \multicolumn{6}{|c|}{ Thermoviscoplastic behaviour } \\
\hline$E(\mathrm{GPa})$ & $\nu(\quad)$ & $A(\mathrm{MPa})$ & $B(\mathrm{MPa})$ & $n(\quad)$ & $\varepsilon_{0}\left(\mathrm{~s}^{1}\right)$ & $C(\quad)$ & $m(\quad)$ \\
\hline 210 & 0.3 & 792 & 510 & 0.34 & 1 & 0.014 & 1.03 \\
\hline
\end{tabular}

Other physical constants

\begin{tabular}{lllll}
\hline$\rho\left(\mathrm{kg} / \mathrm{m}^{3}\right)$ & $\beta($ & $C_{p}(\mathrm{~J} / \mathrm{kg} \mathrm{K})$ & $T_{0}(\mathrm{~K})$ & $T_{m}(\mathrm{~K})$ \\
\hline 2700 & 0.9 & 477 & 293 & 1793
\end{tabular}

\begin{tabular}{llll} 
EOS & & & \\
\hline$c_{0}(\mathrm{~m} / \mathrm{s})$ & $s_{\alpha}$ & $\Gamma_{0}$ & $E_{m}$ \\
\hline 3935 & 1.578 & 1.69 & 0 \\
\hline
\end{tabular}

For AISI 4340, the parameters of the constitutive equation were obtain ed from [36] and are shown in Table 3 together with other physical properties. The Quinney Taylor coefficient which defines the percent age of plastic work converted to heat was taken equal to $\beta=0.9$. The initial temperature $T_{0}$ was set to $293 \mathrm{~K}$ and the melting temperature $T_{m}$ for this alloy is $775 \mathrm{~K}$. Moreover, the model took into account the re lationship between pressure, mass density, and internal energy densi ty/temperature by Mie Gruneisen EOS with the assumptions the Linear Us Up Hugoniot (more details of the expression can be found in [30]).

$p\left(\bar{\varepsilon}^{p}, \dot{\bar{\varepsilon}}^{p}, T\right) \quad \frac{\rho_{0} c_{0}^{2} \eta}{(1-s \eta)^{2}}\left(1-\frac{\Gamma_{0} \eta}{2}\right)+\Gamma_{0} \rho_{0} E_{m}$

where $\rho_{0}$ is the reference density, $c_{0}$ is the speed of sound in the mate rial, $\eta$ is the nominal volumetric compressive strain (expressed as $\eta=$ $1-\rho_{0} / \rho$ being $\rho$ the current density), $\Gamma_{0}$ is a material constant, $E_{m}$ is the internal energy per unit mass, and $s_{\alpha}$ is a constant in Mie Grüneisen hydrodynamic equation of state (Linear Us Up Hugoniot form). The ma terial parameters of EOS are taken from [17], Table 3.

\subsubsection{Full Metal Jacket (FMJ) bullet}

The projectile $9 \mathrm{~mm}$ FMJ bullet, weighting about $8 \mathrm{~g}$, and its FE model are shown in Fig. 3. The dimensions shown are specified accord ing to the STANAG 4090 Ed.2 [25]. The bullet has two components: a copper jacket and a lead core. Seven hundred and fifty two hexahedral elements were used in discretizing the FMJ bullet. The brass jacket material was further modeled by Johnson Cook model and damage ini tiation criterion (Table 7) while the lead material was assumed as elastic with EOS (Table 4) [12].

\subsection{Aramid composite plates}

The most important element of the combat helmet is the shell main ly based on Kevlar composite plates. As it was explained previously first ly a simple model of a plate was developed prior to simulate the more complex geometry of the real helmet. The mesh and the user subroutine were checked in the simple case of the plate and the results were cali brated with experimental data obtained from plate impact. The same type of mesh and laminate configuration were used to model the helmet.

The laminates constituting the plate and the helmet were meshed with C3D6 elements (6 node linear triangular prism) [31] with an ele ment size equal to $4 \mathrm{~mm}$. The FE model of the plates is limited to 4 layers in order to reduce computational cost and increase computational effi ciency [18].

The behaviour of the Kevlar composite was modeled through the in troduction of a user subroutine VUMAT assuming elastic behaviour up to failure. Failure was predicted using the Hashin failure criteria [32] im plemented in a VUMAT user subroutine, modified by Tan et al. [18]. Nu merous studies on low and high velocity impact have demonstrated the accuracy of Hashin failure criteria to model the dynamic behaviour of woven composites, see for example [17 20,22,33]. The failure modes are presented below:

For fiber failure,

1st failure index: tensile fiber 1 mode, $\sigma_{11}>0$

$d_{1 t}\left(\frac{\sigma_{11}}{X_{1 T}}\right)^{2}+\left(\frac{\tau_{12}}{S_{12}}\right)^{2}+\left(\frac{\tau_{13}}{S_{13}}\right)^{2}$

2nd failure index: compressive fiber 1 mode, $\sigma_{11}<0$

$d_{1 C}\left(\frac{\sigma_{11}}{X_{1 C}}\right)^{2}+\left(\frac{\tau_{12}}{S_{12}}\right)^{2}+\left(\frac{\tau_{13}}{S_{13}}\right)^{2}$

3rd failure index: tensile fiber 2 mode, $\boldsymbol{\sigma}_{22}>0$

$d_{2 t}\left(\frac{\sigma_{22}}{X_{2 T}}\right)^{2}+\left(\frac{\tau_{12}}{S_{12}}\right)^{2}+\left(\frac{\tau_{23}}{S_{23}}\right)^{2}$

Table 4

Mechanical properties of cartridge brass and lead core [12].

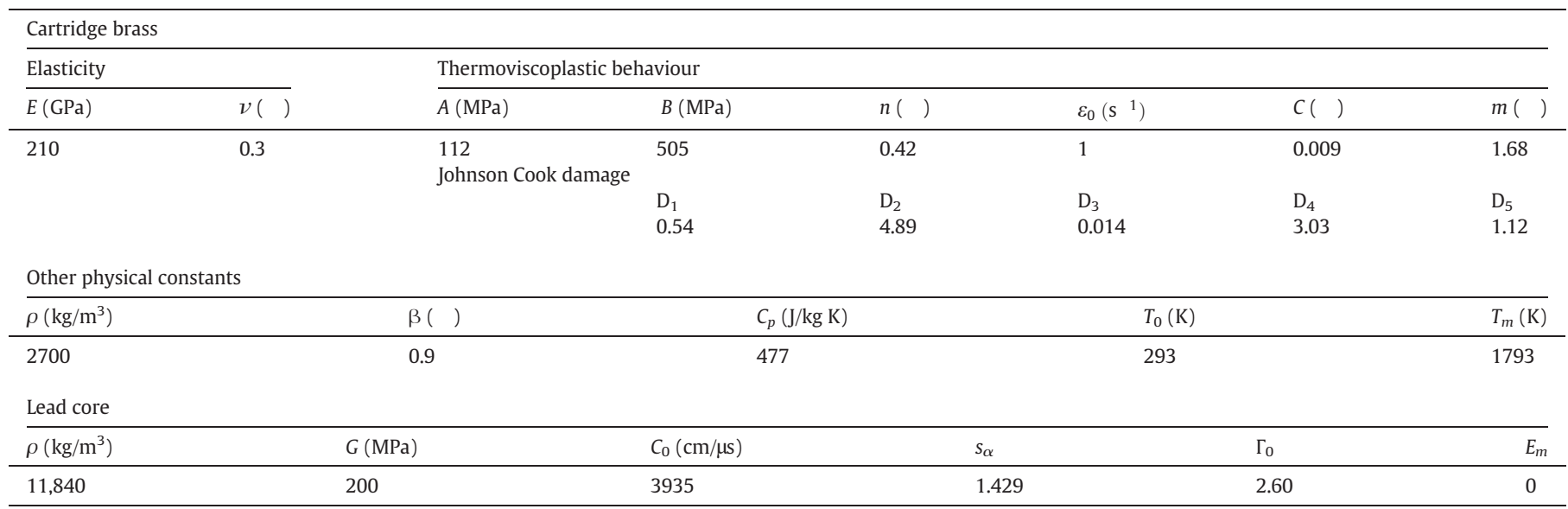


Table 5

Failure properties of Kevlar [18,34].

\begin{tabular}{lllllllll}
\hline $\begin{array}{l}X_{1 T} \\
(\mathrm{MPa})\end{array}$ & $\begin{array}{l}X_{1 C} \\
(\mathrm{MPa})\end{array}$ & $\begin{array}{l}X_{2 T} \\
(\mathrm{MPa})\end{array}$ & $\begin{array}{l}X_{2 C} \\
(\mathrm{MPa})\end{array}$ & $\begin{array}{l}X_{3 T} \\
(\mathrm{MPa})\end{array}$ & $\begin{array}{l}X_{3 C} \\
(\mathrm{MPa})\end{array}$ & $\begin{array}{l}S_{12} \\
(\mathrm{MPa})\end{array}$ & $\begin{array}{l}S_{13} \\
(\mathrm{MPa})\end{array}$ & $\begin{array}{l}S_{23} \\
(\mathrm{MPa})\end{array}$ \\
\hline 555 & 555 & 555 & 555 & 1050 & 1050 & 77 & 1060 & 1086 \\
\hline
\end{tabular}

4th failure index: tensile compressive 2 mode, $\boldsymbol{\sigma}_{22}<0$

$d_{2 C}\left(\frac{\sigma_{22}}{X_{2 C}}\right)^{2}+\left(\frac{\tau_{12}}{S_{12}}\right)^{2}+\left(\frac{\tau_{23}}{S_{23}}\right)^{2}$

For matrix failure,

4th failure index: tensile matrix mode, $\boldsymbol{\sigma}_{33}>0$

$d_{m t}\left(\frac{\sigma_{33}}{X_{3 T}}\right)^{2}+\left(\frac{\tau_{12}}{S_{12}}\right)^{2}+\left(\frac{\tau_{13}}{S_{13}}\right)^{2}+\left(\frac{\tau_{23}}{S_{23}}\right)^{2}$

5th failure index: compressive matrix mode, $\boldsymbol{\sigma}_{33}>0$

$d_{m c}\left(\frac{\sigma_{33}}{X_{3 C}}\right)^{2}+\left(\frac{\tau_{12}}{S_{12}}\right)^{2}+\left(\frac{\tau_{13}}{S_{13}}\right)^{2}+\left(\frac{\tau_{23}}{S_{23}}\right)^{2}$

where $\sigma_{11}, \sigma_{22}$, and $\sigma_{33}$, are the stresses in longitudinal, transverse and through thickness direction respectively; $\sigma_{12}, \sigma_{23}$, and $\sigma_{13}$, are the shear stresses; $X_{1 T}$ and $X_{2 T}$ are the tensile strengths in warp and weft direc tions; $S_{12}, S_{13}$, and $S_{23}$, are the transverse shear strengths. Failure occurs when any damage variable $\left(d_{i j}\right)$ reaches the value 1 .

The reduction of elastic properties could lead to distorted elements involving numerical problems, thus the model requires the use of an el ement erosion criterion. The stresses on a damaged element drop to values close to zero while large deformations appear. These elements do not contribute to the strength or the stiffness of the plate, but they can cause lack of convergence during simulation and instability prob lems. The erosion criterion based on maximum strain criteria, was im plemented in the VUMAT subroutine to remove the distorted elements. After each time increment the longitudinal strains $\left(\varepsilon_{11}, \varepsilon_{22}\right.$ and $\varepsilon_{33}$ ) were evaluated, and the element was removed if one of the strains reached a critical value. The strains used in the erosion criterion were $\varepsilon_{11}^{\max }=1, \varepsilon_{22}^{\max }=1$ and $\varepsilon_{33}^{\max }=1$, high enough to prevent the de letion of elements that contribute to the stiffness and strength of the tar get. Thus, numerical problems were avoided and at the same time only strongly damaged elements were deleted retaining resistant elements.

The parameters for Hashin model were already used by the authors in Tan et al. [18] and obtained from Gong et al. [34], Table 5.

\subsection{Roma Plastilina No. 1}

The standard NIJ STD 0106.01 involves the use of clay for trauma evaluation. Thus, the simulation of the tests corresponding to this stan dard should include modeling of the clay. The material used in the ex perimental work is the commercial clay soft Roma Plastilina No. 1 color grey green. Hernandez et al. [31] carried out experimental tests in order to determine the material parameters associated with a given constitutive model. The characterization methodology posed as an in verse problem of Drop Test was formulated and solved as an optimiza tion procedure. Hernandez et al. [31] proposed Eq. (4) in order to use Roma Plastilina No. 1 in dynamic problems.

$\bar{\sigma} \quad k\left(\bar{\varepsilon}^{p}\right)^{n}\left(\frac{\dot{\bar{\varepsilon}}^{p}}{\dot{\bar{\varepsilon}}_{0}}\right)^{m}$

where $k=0.202$ and is the material constant; $n=0.010$ and is the strain hardening coefficient, $m=0.101$ and is the strain rate sensitivity coeffi cient and $\dot{\bar{\varepsilon}}_{0}$ is the initial strain rate usually set to 1 . Other material prop erties are: density $\rho=1529 \mathrm{~kg} / \mathrm{m}^{3}$, Young Modulus $E=4.92 \mathrm{MPa}$ and Poisson coefficient $\nu=0.49$ obtained from $[31,37]$.

\subsection{Modeling composite plates and validation}

The simple model reproducing a composite plate was used to check the proper definition of parameters such as mesh size, erosion criteria and constitutive model parameters through the comparison between numerical predictions and experimental results.

The numerical models were defined according to the standards in order to be validated through the comparison with the experimental data. Firstly, a numerical model was developed according to STANAG 2920 protocol, which consisted of the plate and FSP projectile. The plate was fixed along all the edges and the friction between the target and the FSP projectile was 0.3.

The numerical evolution of penetration of projectile FSP is shown in Fig. 5 for an interval of $050 \mu$ s. It can be observed that the FSP was stopped around $t=40 \mu$ s. This is consistent with the evolution of the impact velocity of the FSP (Fig. 5) and with the experimental result of the $\mathrm{V}_{50}$.

Secondly, the NIJ STD 0106.01 protocol was also taken into account for model verification. The numerical model consisted of the plate, the FMJ projectile and the Plastilina Roma No. 1 as backing material. The plate was fixed along all the edges, the friction between the target and the FMJ projectile was 0.3 while the coefficient of friction between the bullet and Roma Plastilina No. 1 was equal to 0.193 .

The evolution of penetration process in this case is shown in Fig. 6 .
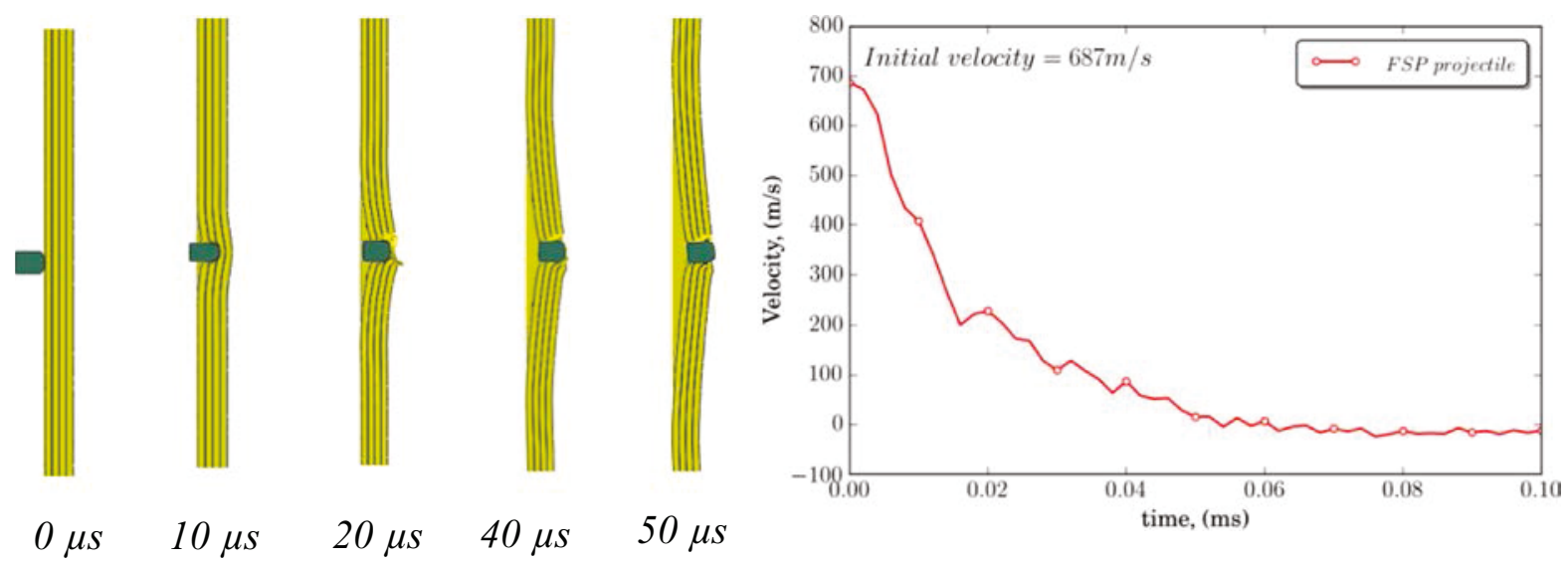

Fig. 5. Evolution of impact process on aramid composite plates for STANAG 2920 protocol. 


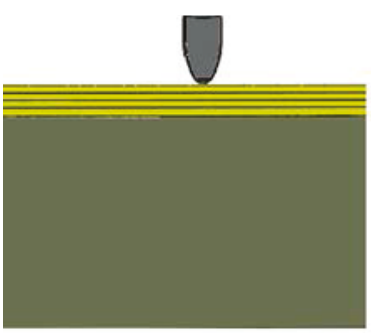

$t=0 \mu s$

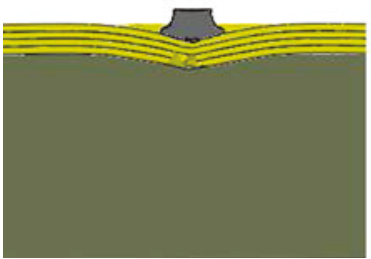

$t=30 \mu \mathrm{s}$

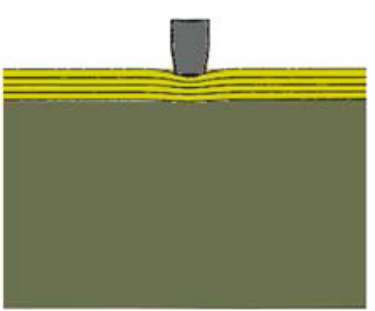

$t=10 \mu \mathrm{s}$

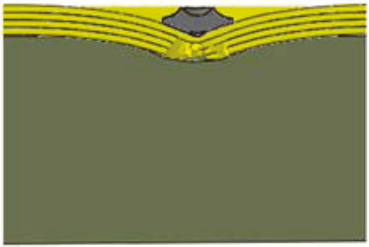

$t=50 \mu \mathrm{s}$

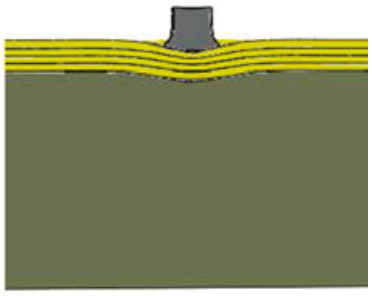

$t=20 \mu s$

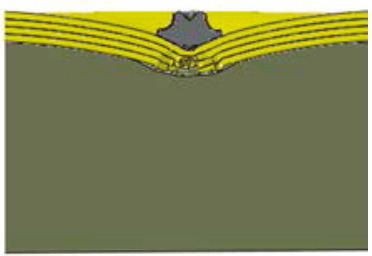

$t=78 \mu \mathrm{s}$

Fig. 6. Evolution of impact process on aramid composite plates for NIJ-STD-0106 protocol.

The calculated BFD was around $20 \mathrm{~mm}$ for impact velocity $\mathrm{V}_{0}=$ $425 \mathrm{~m} / \mathrm{s}$. The back face deformations are in agreement with the experi mental values ( $21.8 \pm 1.35 \mathrm{~mm}$ ); the deformations were slightly lower than experimental values. These values were similar to BFD obtained by Colakoglu et al. [29], $21.6 \mathrm{~mm}$. The difference between the values of this work and those in the literature was due to the different areal density of aramid composite used $\left(9.28 \mathrm{~kg} / \mathrm{m}^{2}\right.$ in [29] and $8.86 \mathrm{~kg} / \mathrm{m}^{2}$ in the pres ent work).

The results showed good accuracy and correct calibration of the nu merical parameters used for aramid composite plates, FSP and FMJ pro jectiles and Plastilina No. 1

\subsection{Modeling complete combat helmet}

The combat helmet was modeled including the considering both the composite shell and the inner protective foams. Also the headform and the clay used in the experiments were reproduced in the model. The im pact of FSP projectiles according to STANAG 2920 protocol were modeled and compared to experimental work in order to analyze the ability of the model to be used as a design tool.

\subsubsection{Combat helmet shell}

The combat helmet shell analyzed in this work has been recently de veloped by the company FECSA [26]. The helmet has notable differences respect to other historical combat helmet described in the literature as the helmets PASGT or ACH; the main geometric difference is the lateral sides, there is no curve change in the zone of ear protection. The design should allow the location of auxiliary systems for communication. Moreover, the density areal is low, $8.86 \mathrm{~kg} / \mathrm{m}^{2}$, while other helmet designs, as PASGT or $\mathrm{ACH}$, have values of areal density around $9.28 \mathrm{~kg} / \mathrm{m}^{2}$ [29].

The FE helmet model (Fig. 7) has been modeled with 4 layers, being the same scheme previously validated for the model of composite plates described in Section 3.1.

\subsubsection{Pad system}

The inner foams were modeled using the Low Density Foam material model available in ABAQUS which is intended for low density, highly compressible elastomeric foams with significant rate sensitive behav iour, such as polyurethane foam. The model requires as input the stress strain response of the material for both uniaxial tension and uni axial compression tests. Rate dependent behaviour is specified by pro viding the uniaxial stress strain curves for different values of nominal strain rates [30]. The mechanical behaviour of the foam padding was ob tained from literature [18]. The mesh of foams was 105,628 hexahedral C3D8R with average size 4 mm, Fig. 6.

\subsubsection{Dummy headform}

The ballistic dummy headform was constructed according to the di mensions specified in the NIJ Standard [24] being the dummy used in the experimental tests, Fig. 7. The headform material was aluminum, and the clay embedded in the head form was Roma Plastilina No. 1. The assembly of different components is illustrated in Fig. 8.
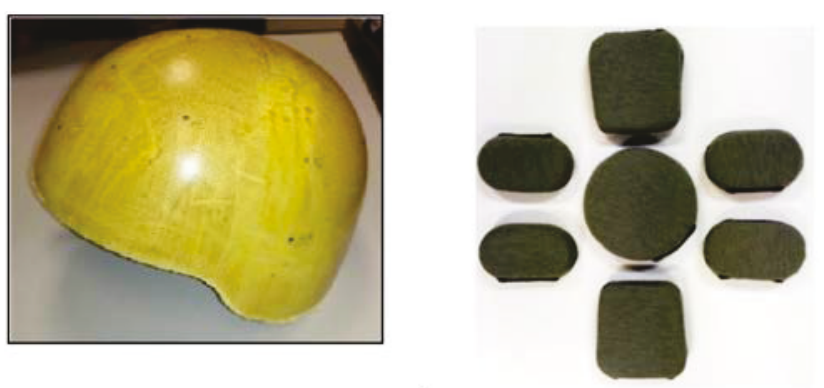

a)
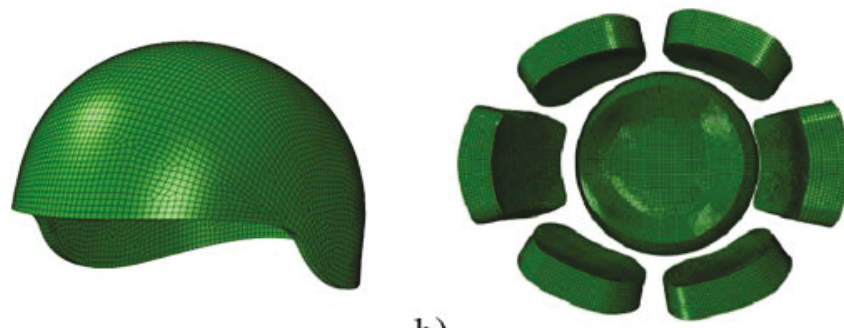

b)

Fig. 7. a) Experimental and b) numerical model of combat helmet shell and interior foams. 

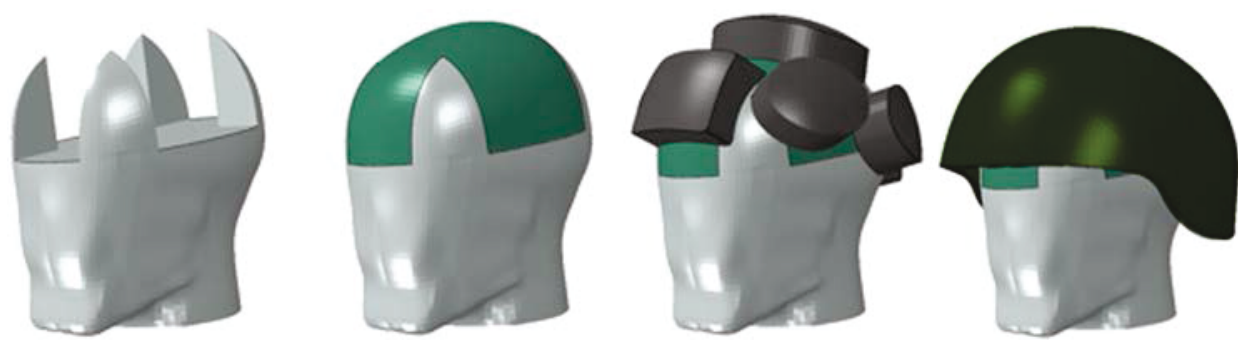

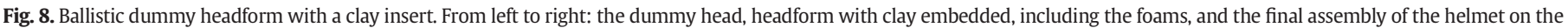
dummy headform.

Two models were developed according to the standards considered in the analysis. The first one consisted of the combat helmet shell and the FSP projectile and was used to obtain the V50 velocity in the case of frontal impact. The second model was developed with the aim of cal culating the back face deformation (BFD) in five positions of impacts: top, frontal, both laterals and rear. This model is shown in Fig. 7.

The contact between the projectile and the helmet and headform components was defined with penalty contact algorithm and hard con tact model with a frictional coefficient $\mu$ equal to 0.3 between steel and composite. For the second model, the coefficient of friction between the FMJ projectile and Roma Plastilina No. 1 was equal to 0.193 according to [31].

\section{Results and discussion}

In this section the numerical results obtained with the model of the real helmet are presented and compared with experimental work. The
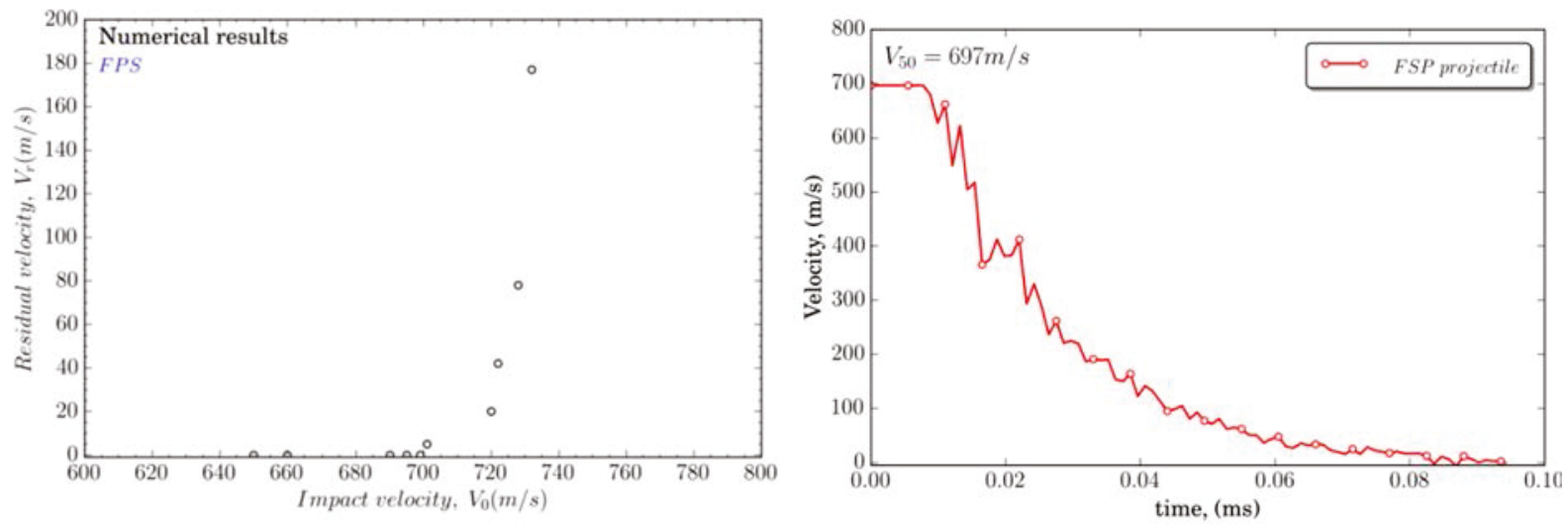

a)

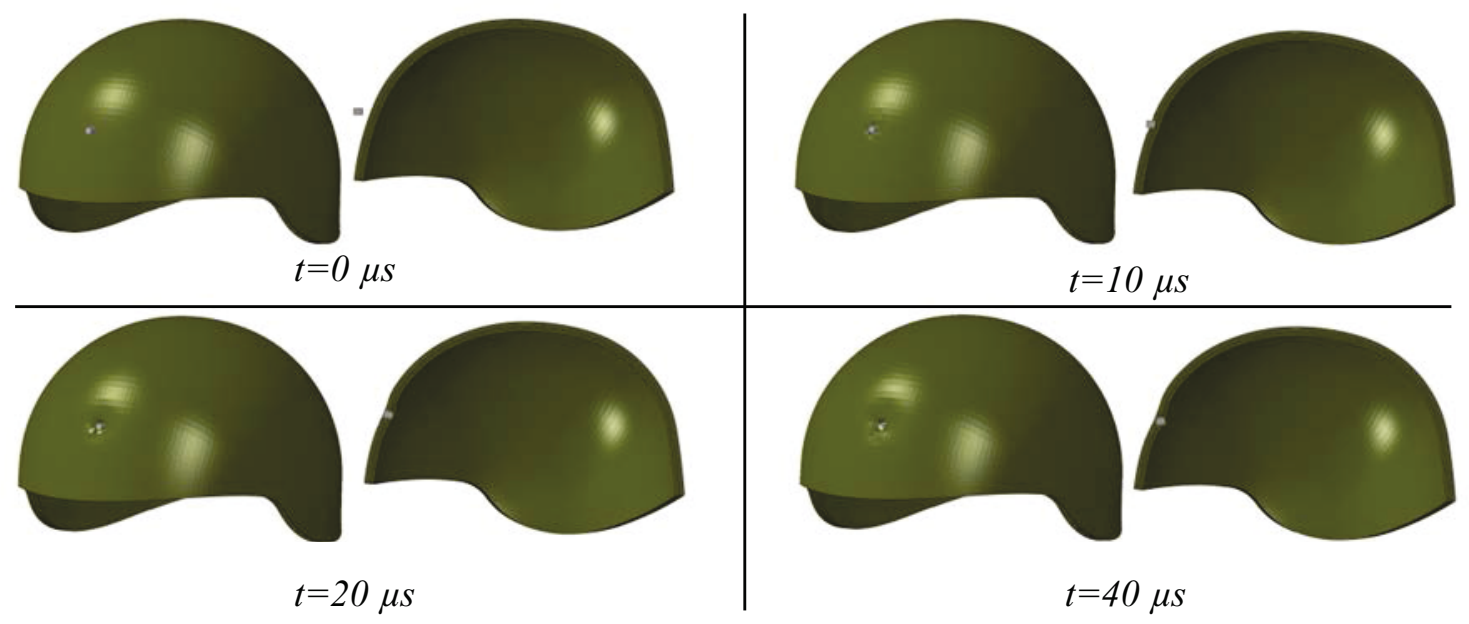

b)

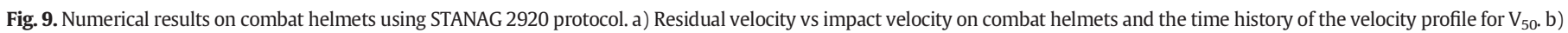
Evolution of impact process on combat helmets. 
Table 6

Experimental and numerical tests in order to obtain the V50 velocity.

\begin{tabular}{|c|c|c|c|}
\hline \multirow[b]{2}{*}{ No. of test } & \multirow[b]{2}{*}{ Impact velocity $(\mathrm{m} / \mathrm{s})$} & \multirow{2}{*}{$\frac{\text { Experimental results }}{\text { Perforation }}$} & \multirow{2}{*}{$\frac{\text { Numerical results }}{\text { Residual velocity }(\mathrm{m} / \mathrm{s})}$} \\
\hline & & & \\
\hline 1 & 663.6 & Partial & - \\
\hline 2 & 683.2 & Partial & - \\
\hline 3 & 689.7 & Partial & - \\
\hline 4 & 697.0 & Partial & - \\
\hline 5 & 701.1 & Complete & Very low $(\sim 0)$ \\
\hline 6 & 701.7 & Complete & Very low $(\sim 0)$ \\
\hline 7 & 718.5 & Complete & 20 \\
\hline 8 & 732.0 & Complete & 177 \\
\hline
\end{tabular}

validity of numerical predictions is evaluated in terms of $V_{50}$, trauma and projectile deformation.

\subsection{Prediction of $V_{50}$}

Numerical simulations were carried out with the aim of predicting the $V_{50}$ velocity with FSP which was found to be around $700 \mathrm{~m} / \mathrm{s}$. The evolution of FSP with time, is plotted in Fig. 9a). In this figure it is ob served that the bullet kept moving on until $t=0.08 \mathrm{~ms}$. The numerical process of the impact is shown in Fig. 9b) where the FSP was stopped by combat helmet shell at velocity $\mathrm{V}_{50}=700 \mathrm{~m} / \mathrm{s}$.

Concerning experimental testing, eight shoots in different places of the helmet were performed covering a wide range of impact velocities $\left(663.6 \mathrm{~m} / \mathrm{s} \leq \mathrm{V}_{0} \leq 732 \mathrm{~m} / \mathrm{s}\right.$ ) (the detail of the values obtained in the tests is included in Table 6 ), resulting in an experimental $V_{50}$ of $697 \mathrm{~m} / \mathrm{s}$ (higher than calculated by other researchers: $680 \mathrm{~m} / \mathrm{s}$ in [17], $660 \mathrm{~m} / \mathrm{s}$ in [38]).

The numerical $\mathrm{V}_{50}$ predicted was also in close agreement with the value obtained from the ballistic test on plates since the combat helmet and the laminate had the same thickness; therefore, both offering sim ilar ballistic resistance.

\subsection{Prediction of trauma}

The analysis of BFD was carried out according to NIJ STD 0106.01 protocol; five shots (front, sides, rear and top of the combat helmet) were conducted with $9 \mathrm{~mm}$ full metal jacket bullet, with nominal mass of $8.0 \mathrm{~g}$ ( 124 grain) and velocity of $425 \pm 15 \mathrm{~m} / \mathrm{s}$.

The results obtained from the simulation in the case of front impact are shown in Fig. 10.

The clay deformation carried out after time $0.36 \mathrm{~ms}$, the maximum value of BFD measured at the clay was reached at time $0.6 \mathrm{~ms}$ being the final permanent deformation value observed after the impact event. Similar behaviour was also reported by Li et al. [8].

Fig. 11 shows the maximum values for the different cases of impact considered. Moreover, in this figure, the simulation results are further il lustrated by plotting the time history of the BFD of the point at the im pact area exhibiting the maximum BFD. The recorded trauma was similar for both frontal and top impacts showing almost the same curve slope. This similar performance was also observed in left and right side lateral impacts. The maximum BFD values were: $10.1 \mathrm{~mm}$ at the frontal zone, $9.67 \mathrm{~mm}$ at the rear area, $10.33 \mathrm{~mm}$ at the top, 5.35 at the right side lateral $\mathrm{mm}$ and $5.13 \mathrm{~mm}$ at lateral side lateral, respec tively. The values were similar to those observed after the experimental tests.

Table 7 summarizes the experimental and numerical values of BFD, the maximum error obtained was around $10 \%$ for all impacts. The max imum value obtained was $12 \mathrm{~mm}$ in the experimental impact at the front of the helmet. This value was lower than $25 \mathrm{~mm}$ which is consid ered the threshold established in the standard [1]. The BFD obtained in frontal impact is twice than that induced in side lateral impacts.

The values of BFD were in general lower than those obtained by other authors when testing combat helmets. Li et al. [8] obtained higher BFD values for frontal and top impacts, $16.56 \mathrm{~mm}$ and $11.39 \mathrm{~mm}$, re spectively. However, the BFD for right side lateral impact was $5.05 \mathrm{~mm}$, which is slightly lower than our experimental test.

\subsection{Projectile and shell deformation}

The extension of the deformed zone obtained from experiments and simulations is presented in Fig. 12. Penetration of the projectile into the helmet did not occur in any tests performed and the extension and shape of the deformed area was similar for experiments and numerical simulations.

The deformed bullets display a classical mushroom shape with di ameters of $17.94 \mathrm{~mm}, 21.36 \mathrm{~mm}, 17.69 \mathrm{~mm}$ for top, frontal and rear

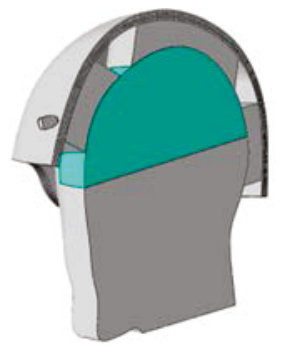

$t=0 \mathrm{~ms}$

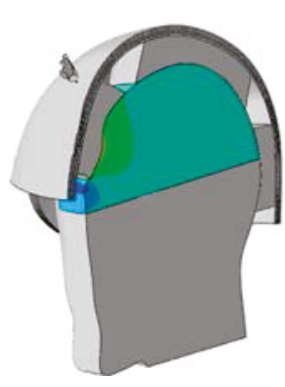

$t=0.6 \mathrm{~ms}$

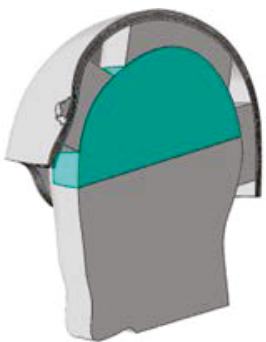

$t=0.05 \mathrm{~ms}$

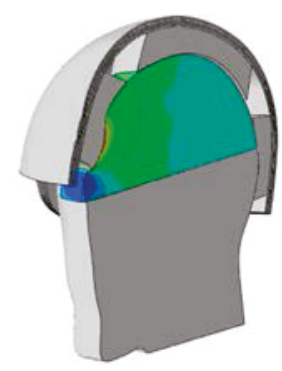

$t=1.24 \mathrm{~ms}$

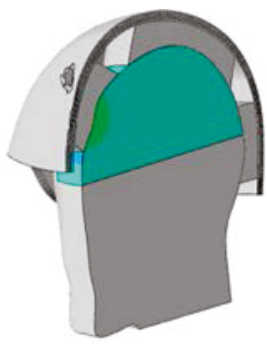

$t=0.36 \mathrm{~ms}$

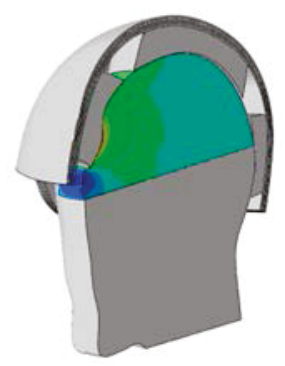

$B F D(\mathrm{~mm})$

$$
t=1.5 \mathrm{~ms}
$$

Fig. 10. Sequence of the front impact event showing the deformation of the clay. 

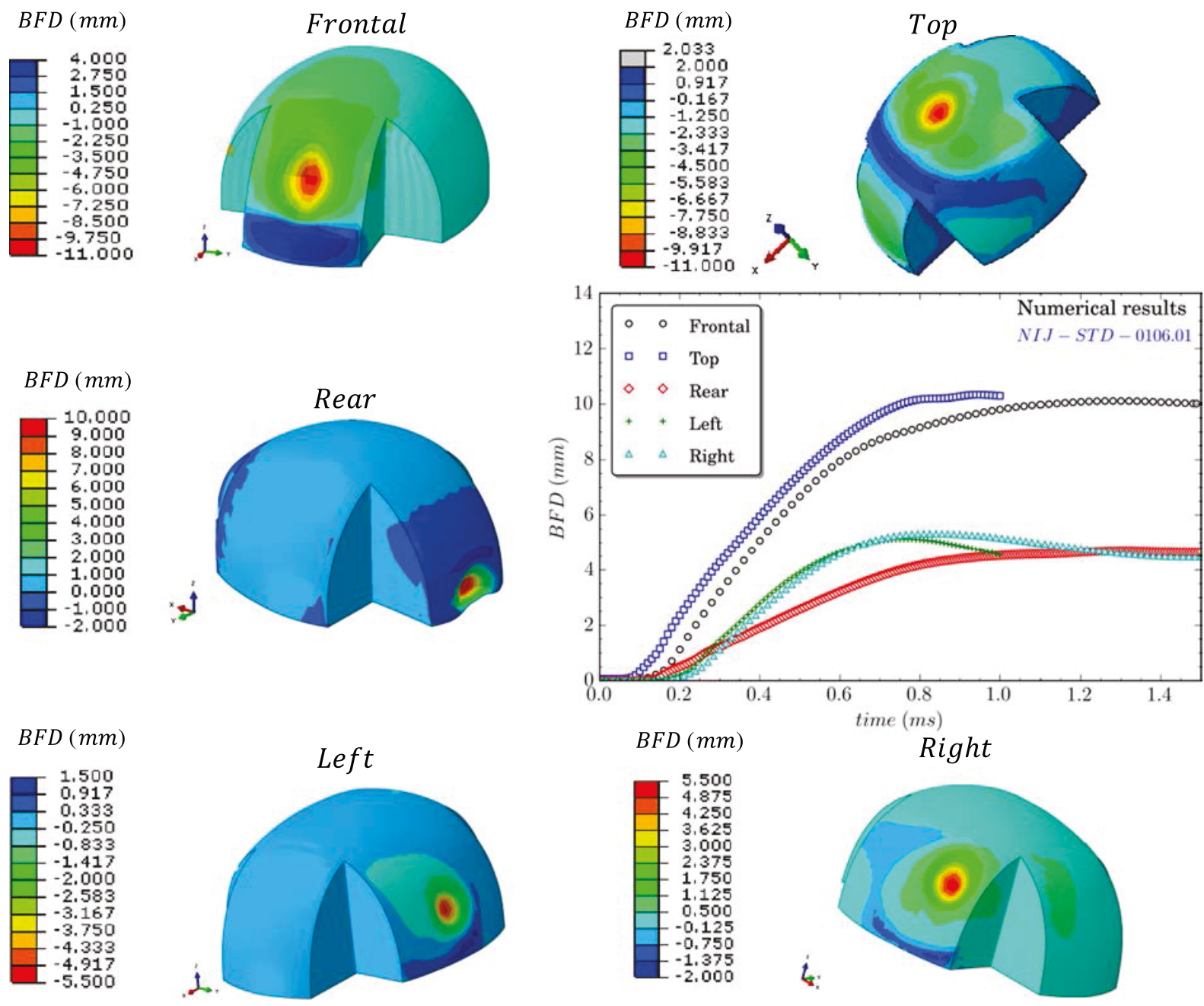

Fig. 11. BFD in the dummy head/clay and the time history of the BFD for the different impacts.

impacts, respectively, in the final, permanently deformed state, which was closed to the experimental finding of $15.94 \mathrm{~mm}, 21.41 \mathrm{~mm}$ and $19.99 \mathrm{~mm}$ for top, frontal and rear impacts, respectively. The experi mental and numerical final stage of deformed bullets (shape and size) is shown in Fig. 12 showing good accuracy of the numerical predictions.

\section{Conclusions}

In this paper, a numerical finite element model was developed in order to predict the response of a combat helmet subjected to ballistic impact. The experimental work was carried out to calibrate the material properties and validate the ballistic performance of the real helmet presented.

Table 7

Experimental and numerical comparison of back face deformation (BFD) for the combat helmet.

\begin{tabular}{lllll}
\hline Position & Impact velocity $(\mathrm{m} / \mathrm{s})$ & Exp. BFD $(\mathrm{mm})$ & Num. BFD $(\mathrm{mm})$ & Error $(\%)$ \\
\hline Front & 427.3 & 12 & 10.1 & 15.8 \\
Right & 420.7 & 6 & 5.35 & 10.8 \\
Back & 419.7 & 9 & 9.67 & 7.44 \\
Left & 433.8 & 6 & 5.13 & 14.5 \\
Top & 421.5 & 11 & 10.3 & 6.3 \\
& & & Average error (\%) & 10.9 \\
\hline
\end{tabular}

Finite element modeling for impact on plates and on combat helmet is presented. Firstly, a simplified model of a plate was developed in order to check mesh and user subroutine configuration. The model pa rameters were calibrated through experimental tests on plates per formed according to STANAG 2920 and NIJ STD 0106.01. The simulations accurately reproduced experiments, showing good agree ment with ballistics tests involving normalized projectile.

Secondly the numerical model of the complete helmet under ballis tic impact was developed. The model was used to calculate the ballistic limit of the combat helmet, providing a $V_{50}$ velocity of $697.0 \mathrm{~m} / \mathrm{s}$. Nu merical results were compared with real impact tests showing the accu racy of the model and its suitability to be used as a designing tool. Despite the helmet shell configuration presented a reduced areal densi ty when compared with other designs referred in the literature, results for the BFD and the $\mathrm{V}_{50}$ velocity were improved.

The results of this work have demonstrated that the helmet evaluat ed in this study has the potential to meet the requirements established by manufacturers for helmet performance against a specific set of ballis tic treats. Also the modeling methodology proposed has showed its abil ity to be used as a tool design.

\section{Acknowledgments}

The authors are indebted to the Spanish company FECSA for provid ing the helmets tested in the paper. 

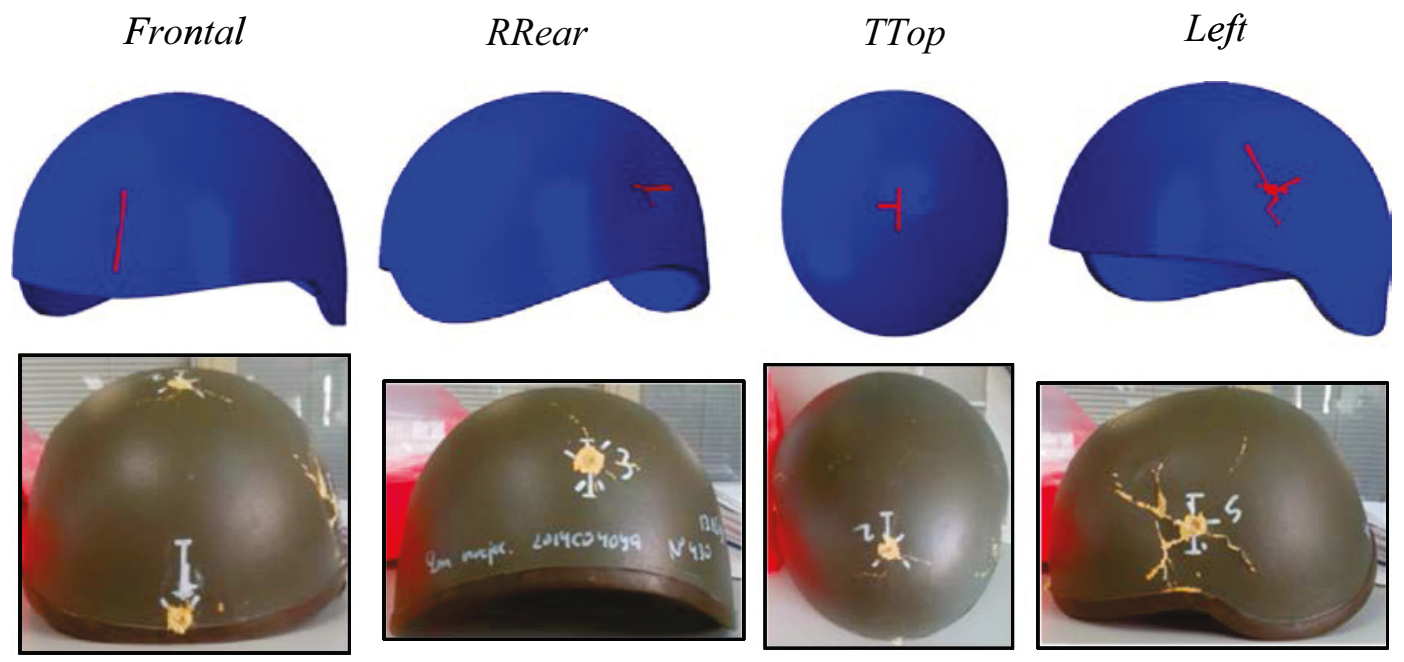

a)

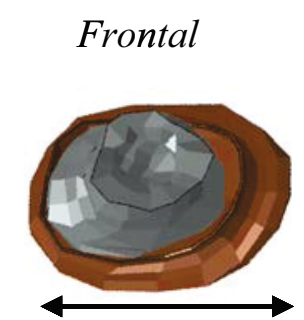

$21.41 \mathrm{~mm}$

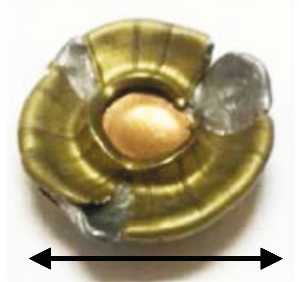

$21.36 \mathrm{~mm}$
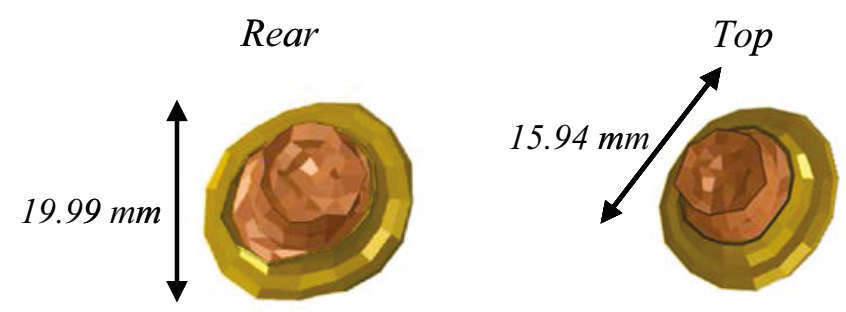

$17.69 \mathrm{~mm}$
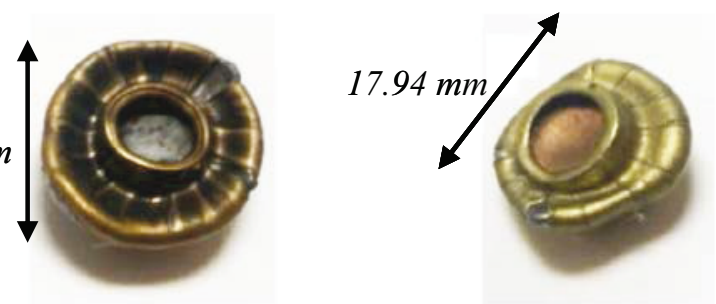

b)

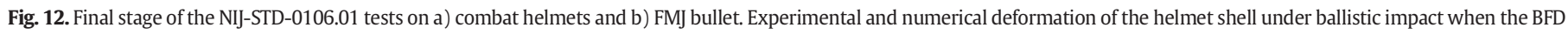

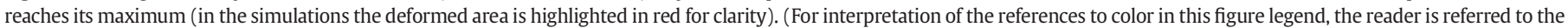
web version of this article.)

The authors acknowledge the Ministry of Economy and Competi tiveness of Spain and FEDER program under the Project RTC 2015 38878 for the financial support of the work.

\section{References}

[1] Council N R, Review of Department of Defense Test Protocols for Combat Helmets, 2014.

[2] H. López-Gálvez, M. Rodriguez-Millán, N. Feito, H. Miguelez, A method for inter-yarn friction coefficient calculation for plain wave of aramid fibers, Mech. Res. Commun. 74 (2016) 52-56.

[3] A. Majumdar, B.S. Butola, A. Srivastava, An analysis of deformation and energy absorption modes of shear thickening fluid treated Kevlar fabrics as soft body armour materials, Mater. Des. 51 (2013) 148-153.

[4] A.A. Ramadhan, A.R. Abu Talib, A.S. Mohd Rafie, R. Zahari, High velocity impact response of Kevlar-29/epoxy and 6061-T6 aluminum laminated panels, Mater. Des. 43 (2013) 307-321.

[5] P.K. Pinnoji, P. Mahajan, Analysis of impact-induced damage and delamination in the composite shell of a helmet, Mater. Des. 31 (2010) 3716-3723.

[6] M. Grujicic, W.C. Bell, B. Pandurangan, T. He, Blast-wave impact-mitigation capability of polyurea when used as helmet suspension-pad material, Mater. Des. 31 (2010) 4050-4065.

[7] Y. Zhou, Development of Lightweight Soft Body ArmourPhD Dissertation University of Manchester, Department of Engineering and Physical Sciences, 2013.
[8] Y.Q. Li, X.G. Li, X.L. Gao, Modeling of advanced combat helmet under ballistic impact, J. Appl. Mech. 82 (2015) 1110041-1110049.

[9] A.W. Carroll, C.A. Soderstrom, A new nonpenetrating ballistic injury, Ann. Surg. 188 (1978) 753-757.

[10] J.C. Sarron, J.P. Caillou, J. Da Cunha, J.C. Allain, A. Tramecon, Consequences of nonpenetrating projectile impact on a protected head: study of rear effects of protections, J. Trauma 49 (2000) 923-929.

[11] K.A. Rafaels, H.C. Cutcliffe, R.S. Salzar, M. Davis, B. Boggess, B. Bush, R. Harris, M.S Rountree, E. Sanderson, S. Campman, S. Koch, C.R. Dale Bass, Injuries of the head from backface deformation of ballistic protective helmets under ballistic impact, J. Forensic Sci. 60 (2015) 219-225.

[12] K.M. Tse, Development of a Realistic Finite Element Model of Human Head and its Applications to Head InjuriesPhD Dissertation National University of Singapore, Department of Mechanical Engineering, 2013.

[13] L. Tan, F.S. Bin Chew, K.M. Tse, V.B.C. Tan, H.P. Lee, Impact of complex blast waves on the human head: a computational study, Int. J. Numer. Methods Biomed. Eng. 1-30 (2014).

[14] D. Singh, D.S. Cronin, T.N. Haladuick, Head and brain response to blast using sagittal and transverse finite element models, Int. J. Numer. Methods Biomed. Eng. 30 (2014) 470-489.

[15] D. Jenson, V.U. Unnikrishnan, Energy dissipation of nanocomposite based helmets for blast-induced traumatic brain injury mitigation, Compos. Struct. 121 (2015) 211-216.

[16] D.M. Hisley, J.C. Gurganus, A.W. Drysdale, Experimental methodology using digital image correlation to assess ballistic helmet blunt trauma, ASME J. Appl. Mech. 78 (2011) 051022. 
[17] C.Y. Tham, V.B.C. Tan, H.P. Lee, Ballistic impact of a KEVLAR® helmet: experiment and simulations, Int. J. Impact Eng. 35 (2008) 304-318.

[18] L.B. Tan, K.M. Tse, H.P. Lee, V.B.C. Tan, S.P. Lim, Performance of an advanced combat helmet with different interior cushioning systems in ballistic impact: experiments and finite element simulations, Int. J. Impact Eng. 50 (2012) 99-112.

[19] J. van Hoof, D.S. Cronin, M.J. Worswick, K.V. Williams, D. Nandlall, Numerical head and composite helmet models to predict blunt trauma, 19th International Symposium of Ballistics 2001, pp. 921-928.

[20] M. Aare, S. Kleiven, Evaluation of head response to ballistic helmet impacts using the finite element method, Int. J. Impact Eng. 34 (3) (2007) 596-608.

21] Beschussamt Mellrichstadt, Test Protokoll-Nr: 01M072-01, Date: 21.03.2001; 01M073A01, 21.03.2001; 01M079-01, 29.03.2001; 01M085A01, 30.03.2001; 01M085B01, 30.03.2001, Bericht-No: 140-143, Date: 19.03.2001, Beschussamt Mellrichstadt, Lohstrasse 5, 97638 Mellrichstadt, Germany, March 2001.

[22] H.P. Lee, S.W. Gong, Finite element analysis for the evaluation of protective functions of helmets against ballistic impact, Comput. Methods Biomech. Biomed. Engin. 13 (5) (2010) 537-550.

23] K.M. Tse, L.B. Tan, B. Yang, V.B.C. Tan, S.P. Lim, H.P. Lee, Ballistic impacts of a fullmetal jacketed (FMJ) bullet on a validated finite element (FE) model of helmetcushion-head, in: G.R. Liu, Z.W. Guan (Eds.), Proceedings of the 5th International Conference on Computational Methods (ICCM), Cambridge, UK, ScienTech Publisher 2014, pp. 1-8.

[24] National Institute of Justice (NIJ) Standard, NILECJ-STD-0106.00: Ballistic Helmets, U.S.D.o. Justice, Washington, DC, US, 1975.

[25] J.H. Eriksen, STANAG 2920 Ed 2 Ballistic Test Method for Personal Armor Materials and Combat Clothing, second ed. NATO PfP, 2003.

[26] Fábrica de Confecciones Española S.A, https://www.fecsa.net2016 (accessed 21.05.16).

[27] NME-2786 Requisitos técnicos para la homologación del casco de Combate, 2013.
[28] J. van Hoof, Modelling Impact Induced Delamination in Composite Materials, Carleton University, Ottawa, 1999.

[29] M. Colakoglu, O. Soykasap, T. Army, T. Özek, Experimental and numerical investigations on the ballistic performance of polymer matrix composites used in armor design, Appl. Compos. Mater. 2 (2007) 1-8.

[30] Dassault Systèmes, Abaqus v6.12 Documentation- ABAQUS Analysis User's Manual, ABAQUS Inc, 2012 (6.12).

[31] C. Hernandez, M.F. Buchely, A. Maranon, Dynamic characterization of Roma Plastilina No. 1 from Drop Test and inverse analysis, Int. J. Mech. Sci. 100 (2015) $158-168$.

[32] Z. Hashin, Failure criteria for unidirectional fiber composites, Trans. ASME J. Appl. Mech. 47 (1980) 329-334.

[33] M. Rodríguez-Millán, C.E. Moreno, M. Marco, C. Santiuste, H. Miguélez, Numerical analysis of the ballistic behaviour of Kevlar composite under impact of doublenosed stepped cylindrical projectiles, J. Reinf. Plast. Compos. 35 (2016) 124-137.

[34] S.W. Gong, H.P. Lee, C. Lu, Computational simulation of the human head response to non-contact impact, Compos. Struct. 86 (2008) 758-770.

[35] G.R. Johnson, W.H. Cook, A constitutive model and data for metals subjected to large strains high strain rates and high temperatures, Proceedings of the Seventh International Symposium on Ballistics, 1983.

[36] T. Ozel, E. Zeren, Finite element modeling the influence of edge roundness on the stress and temperature fields induced by high speed machining, Int. J. Adv. Manuf. Technol. 35 (2007) 255-267.

[37] H. Sofuoglu, J. Rasty, Flow behavior of Plasticine used in physical modeling of metal forming processes, Tribol. Int. 33 (8) (2000) 523-529.

[38] P.M. Cunniff, Decoupled response of textile body armor, Proceedings of the 18th International Symposium on Ballistics, San Antonio, TX, USA 1999, pp. 814-821.

[39] J.S. Lim, J.H. Kim, Ballistic behavior of Heracron ${ }^{\circledR}$-based composites: effect of fiber density and fabrication method, Compos. Interfaces 21 (6) (2014) 543-552. 